\title{
Indicaxanthin, Miraxanthin- $V$, and Hexahydrocurcumin as Potential Erythropoietin Agonist in Silico to Treat Anemia in Chronic Kidney Disease
}

\author{
Yuliana Heri Suselo ${ }^{1 *}$, Muhammad Rizky Kamil ${ }^{2}$, Sri Wulandari ${ }^{3}$, and Dono Indarto ${ }^{4,5}$ \\ ${ }^{1,3,4}$ Physiology Department, Faculty of Medicine, Universitas Sebelas Maret Surakarta, Indonesia \\ Jalan Semarang - Surakarta, Jebres, Kota Surakarta, Jawa Tengah 57126 \\ ${ }^{2}$ Medical Student, Faculty of Medicine, Universitas Sebelas Maret Surakarta \\ ${ }^{5}$ Biomedical Laboratory, Faculty of Medicine, Universitas Sebelas Maret Surakarta \\ *Corresponding author: yulianaheri@ staff.uns.ac.id
}

\begin{abstract}
Background: Anemia is the most frequent complication of Chronic Kidney Disease (CKD) which is mainly caused by erythropoietin (Epo) deficiency. Epo agonist is the drug choice for anemia in CKD but some patients have antibody against Epo agonist. Objectives: This study aimed to identify Indonesian medicinal plants that have an agonist activity to Epo receptor in silico. Method: This was a bioinformatics study with using all Indonesian phytochemicals which were registered in HerbalDB and had the 3-D in PubChem. The Epo-EpoR complexes were used as standard ligand and receptor with the Protein Data Bank code 1CN4. Because Epo and EpoR sizes were bigger than 1,500 Da, the molecules were truncated validated 3 times using AutodockVina 1.1.2. and all phytochemicals were molecularly docked using the same method. Docking results were visualized using PyMOL 1.7.4. Results: Truncated Epo interacted with EpoR in 9 different binding sites with average of binding affinity ranging from -2.6 to $-5.5 \mathrm{kcal} / \mathrm{mol}$. Indicaxanthin, Miraxanthin-V, and Hexahydrocurcumin had lower binding affinity than standard in each binding sites. Similar binding sites to EpoR were founded in Indicaxanthin. Conclusion: Indicaxanthin, Miraxanthin-V, and Hexahydrocurcumin were potential as Epo agonist in silico to treat anemia in CKD.
\end{abstract}

Keywords: Agonist Erythropoietin, Anemia, CKD, Phythochemicals, Molecular Docking

\section{INTRODUCTION}

Anemia is still a major nutrition problem in developing countries. Many South-East Asia countries have higher prevalence of anemia such as Kamboja 63\%, Bangladesh $47 \%$ and Indonesia 44.5\% (WHO, 2015). In Indonesia, the highest prevalence of anemia is found in pregnant women $(48.2 \%)$, followed by elder people (46\%), non pregnant women $(45.7 \%)$, children $(28.1 \%)$, and adult (16.9\%) (Balitbang Kemenkes RI, 2013). In addition, 20-30\% of anemia is caused by chronic diseases including Chronic Kidney Disease (CKD) (Oliveira et al, 2014). The prevalence of anemia in CKD patients can be as high as 58.5\% if CKD patients are treated without dialysis (Cases-Amenos et al., 2014).

The main cause of anemia in CKD is Epo deficiency which is related to decrease of erythrocyte life cycle. Deficiency of iron, vitamin and other factors can also contribute in CKD anemia [4]. As a result, the kidney volume decreases significantly in conjunction with severity of $\mathrm{CKD}$, which decreases more erythropoeietin production. Epo deficiency also inhibits erythrocyte maturation from progenitor cell to normoblast and reticulocyte. Therefore, imature erythrocytes become fragile and lysis (neocytolisis). From microscopic observation, erythrocytes have normocytic and normochromic features that is different from erythrocyte features of iron deficiency anemia (Hayat et al., 2008; Mehdi \& Toto, 2009).

According to National Kidney Foundation (2012), the standard therapy of CKD is iron supplementation, administration of erythropoiesis-stimulating agents (ESAs), and red blood 
cell transfusion. In Indonesia the use of ESA has increased considerably, compared to iron supplementation and blood transfusion (PERNEFRI, 2014). Recombinant Epo and its derivatives (epoetin $\alpha$, epoetin $\beta$, darbopoetin $\alpha$, and methoxy polyethylene glycol-epoetin $\beta$ ) are the most common ESAs to treat CKD anemia (Palmer et al., 2014).

ESAs group still have some limitations due to effectiveness and efficacy. Approximately 5\%-10\% of CKD patients are resistance to ESA (Johnson et al., 2007). In addition, long-term use of ESA in rat model of CKD results in formation of anti-EPO antibodies (Garrido et al., 2012). Administration of ESAs in CKD patients for long time also increases cardiovascular disorders like stroke, venous thromboembolism and red blood cell aplasia (Clemet et al., 2009; Parfrey et al., 2009; Palmer et al., 2010; Macdougall et al., 2014). Because of high cost of ESAs treatment, only 20-30\% of CKD patients receive ESAs prior to dialysis (National Kidney Foundation, 2012; Kim et al., 2016).

Indonesia has $75 \%$ plants species from total 40,000 plants in the world. However, about 9,600 species are just known to have pharmacological effects (Yanuar et al., 2012). So, the diversity of Indonesian plants can be utilized for drug development to treat various human diseases, including CKD anemia. Virtual Screening (VS) is one of the most common method of drug discovery which has some benefits such as faster, effective, and low cost (Tang and Marshal, 2011). Molecular docking is frequently used to screen active compounds in natural resources (Ferreira et al., 2015). Therefore, the purpose of this study was to identify molecularly Indonesian plants that have activity as an agonist for drug development of CKD anemia.

\section{METHOD}

This was a bioinformatics research study using molecular docking method. The 3dimensional structure of erythropoietin receptors (ID: 1CN4) binding to erythropoietin was obtained from Protein Data Bank (http://www.rscb.org/pdb/) as a target protein for screening phytochemicals of Indonesian plants (http://www.herbaldb.farmasi.ui.ac.id). The 3dimensional structure of phytochemicals was obtained from PubChem NCBI (http://pubchem.ncbi.nlm.nih.gov) and met the Lipinski's criteria [20,21]. Software of AutoDock Tools version 1.5.6 (2013), PyRx version 0.8 (2010) and PyMol 1.7.4 was freely downloaded.

\section{Preparation of three-dimensional structure of compound Epo-EpoR as standard}

The Epo-EpoR binding complexes were separated by using AutoDock Tools and the molecular structure of Epo and EpoR was optimized by removing water molecules and adding hydrogen atoms. Due to molecular weight of Epo more than 1,500 Da, the Epo molecule was truncated into 9 parts based on binding sites on EpoR monomer 1 and monomer 2 (Table 1). Each Epo part was validated several times with EpoR until the Root Mean Square Deviation (RMSD) score was <2 $\AA$ (Palmer et al, 2010). Binding energy of truncated Epo-EpoR complexes was then compared to binding energy of the previous study (Syed et al, 1998) 
Table 1 Location of truncated Epo-EpoR interaction

\begin{tabular}{|c|c|}
\hline Location of Epo binding & Truncated Epo residues \\
\hline Lys $^{20}$ & $\mathrm{Ala}^{19}-\mathrm{X}^{\mathrm{n}}-\mathrm{Glu}^{23}$ \\
\hline $\mathrm{Thr}^{44}-\mathrm{X}^{\mathrm{n}}-\mathrm{Asn} 47$ & $\mathrm{Thr}^{44}-\mathrm{X}^{\mathrm{n}}-\mathrm{Phe}^{48}$ \\
\hline $\operatorname{Arg}^{131}$ & Asp ${ }^{131}-X^{n}-I_{1}{ }^{133}$ \\
\hline Lys $^{140}$ dan Arg ${ }^{143}$ & Lys $^{140}-X^{\mathrm{n}}-\operatorname{Arg}^{143}$ \\
\hline $\operatorname{Asn}^{147}$ dan $\operatorname{Arg}^{150}$ & $\operatorname{Asn}^{147}-X^{\mathrm{n}}-\operatorname{Arg}^{150}$ \\
\hline $\mathrm{Asp}^{8}$ & Asp $^{8}$ dan $\operatorname{Ser}^{9}$ \\
\hline $\operatorname{Arg}^{14}$ & $\operatorname{Ser}^{13}-\mathrm{X}^{\mathrm{n}}-\mathrm{Tyr}^{15}$ \\
\hline Lys $^{97}$ dan Ser ${ }^{100}$ & Lys $^{97}-X^{\mathrm{n}}-\mathrm{Ser}^{100}$ \\
\hline $\operatorname{Arg}^{103}$ dan $\operatorname{Ser}^{104}$ & Arg ${ }^{103}-X^{\mathrm{n}}-$ Leu $^{105}$ \\
\hline
\end{tabular}

\section{Docking Analysis of Phytochemicals}

The PyRx applications was used to dock molecularly phytochemicals with EpoR. Phytochemicals should be interacted with residues in the binding sites of EpoR. More negative binding energy of phytochemicals-EpoR interaction was considered as candidates Epo agonist. Pymol software was used to visualize the location of phytochemicals-EpoR interaction and to compare the molecular conformation with the standard.

\section{RESULTS AND DISCUSSION}

There were nine parts of truncated homodimeric Epos (MW= 17,246.74 g/mol) which five truncated Epos were bond to EpoR monomer 1 and the remaining truncated Epos were bond to the EpoR monomer 2 (Table 2). For standard 1, It consisted of $\mathrm{Ala}^{19}-\mathrm{X}^{\mathrm{n}}-\mathrm{Glu}^{23}(\mathrm{MW}=$ $273.78 \mathrm{~g} / \mathrm{mol}$ ) residues which interacted with a residue at $\mathrm{Glu}^{202}$ EpoR and had average of binding energy $(-3.9 \mathrm{kcal} / \mathrm{mol})$. Amino acids $\left(\mathrm{Glu}^{62}, \mathrm{Thr}^{87}, \mathrm{Ala}^{88}\right.$, and $\mathrm{Ser}^{92}$ ) of EpoR interacted with standard 2 Epo (MW=591.70 $\mathrm{g} / \mathrm{mol}$ ) with average binding energy $-5.2 \mathrm{kcal} / \mathrm{mol}$. Interaction between $\mathrm{Asp}^{131}-\mathrm{X}^{\mathrm{n}}$-Ile ${ }^{133}$ residues in standard 3 Epo and $\mathrm{Asp}^{61}$-Glu ${ }^{62}$ residues of EpoR had average binding energy $-3.8 \mathrm{kcal} / \mathrm{mol}$. For standard 4, 5, and 8 truncated Epos had around $500 \mathrm{~g} / \mathrm{mol}$ at $\mathrm{Asp}^{61}$; $\mathrm{Phe}^{93}, \mathrm{Glu}^{117}$, Pro ${ }^{203}$ and $\mathrm{Ser}^{204}$; $\mathrm{Glu}^{34}, \mathrm{Ala}^{88}$, and $\mathrm{Ser}^{91}$ of EpoR respectively which average binding energy was from -5.5 to $-3.8 \mathrm{kcal} / \mathrm{mol}$. Lower molecular weight and higher binding energy were observed in truncated Epos as standard 6, 7, and 9, compared to the molecular weight 4, 5 and 8. These standards interacted with EpoR at His ${ }^{153}$; $\mathrm{Leu}^{33}$ and $\mathrm{Ser}^{92}$; $\mathrm{Glu}^{62}, \mathrm{Ala}^{88}, \mathrm{Asp}^{89}, \mathrm{Ser}^{91}$, and $\mathrm{Ser}^{92}$ respectively except energy binding of standard 9 was lower than that of standard 4 and 8 and was higher than standard 5 .

Table 3 showed that all Epo standards had hydrogen and van der Walls bonds to EpoR except standard 4 and 7 which had only hydrogen bond. Standard 2 had the highest number of hydrogen bond, followed by standard 6 and 9 ( 4 hydrogen bonds), standard 3 with 3 hydrogen bonds and 2 hydrogen bonds in standard 8 . Other standards had only one hydrogen bond. More than or equal 2 van der walls bonds were found in standard 2, standard 5, standard 6 and standard 8 while standar 3 and 9 had only one van der walls bond.

Although we used truncated Epos, these molecules similarly interact with EpoR as reported by Syed and co-workers. However, 1-5 Epo standards have 11 binding sites (Glu ${ }^{60}$, $\mathrm{Asp}^{61}, \mathrm{Glu}^{62}, \mathrm{Thr}^{87}, \mathrm{Ala}^{88}, \mathrm{Ser}^{92}, \mathrm{Phe}^{93}, \mathrm{Glu}^{117}, \mathrm{Glu}^{202}, \mathrm{Pro}^{203}$ and $\mathrm{Ser}^{204}$ ) and lack of His ${ }^{114}$ binding site in monomer 1 of EpoR. A higher similarity of binding sites is also found in standard 6-9 at $\mathrm{Leu}^{33}, \mathrm{Glu}^{62}, \mathrm{Ala}^{88}, \mathrm{Asp}^{89}, \mathrm{Ser}^{91}, \mathrm{Ser}^{92}$, and His ${ }^{153}$ residues (Syed et al, 1998). Therefore, this docking process can be used for model of Epo-EpoR binding complexes. 
Table 2 Biochemical properties of Epo and Docking Results between Epo and EpoR

\begin{tabular}{|c|c|c|c|c|c|}
\hline $\begin{array}{l}\text { Truncated Epos } \\
\text { as Standards }\end{array}$ & $\begin{array}{l}\text { Residues of } \\
\text { Epos } \\
\text { Standards }\end{array}$ & $\begin{array}{l}\text { Molecular } \\
\text { chemical } \\
\text { formulas }\end{array}$ & $\begin{array}{c}\text { Molecular } \\
\text { weight } \\
\text { (g/mol) }\end{array}$ & $\begin{array}{c}\text { Docking } \\
\text { Score } \\
\text { (kcal/mol) }\end{array}$ & $\begin{array}{l}\text { Location binding } \\
\text { interaction of } \\
\text { Epo-EpoR }\end{array}$ \\
\hline $\begin{array}{l}\mathrm{Lys}^{20 *}{ }_{---\mathrm{Glu}^{202}} \\
\text { (Standard 1) }\end{array}$ & $\mathrm{Ala}^{19}-\mathrm{X}^{\mathrm{n}}-\mathrm{Glu}^{23}$ & $\mathrm{C}_{19} \mathrm{H}_{31} \mathrm{~N}_{5} \mathrm{O}_{9}$ & 473,48 & $-3,9$ & $\begin{array}{l}\mathrm{Asn}^{116}, \mathrm{Glu}^{117}, \mathrm{Glu}^{202}, \\
\mathrm{Pro}^{203}, \mathrm{Ser}^{204}\end{array}$ \\
\hline 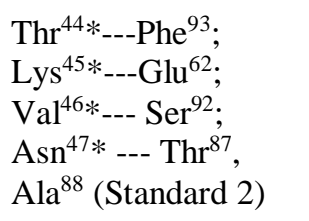 & $\mathrm{Thr}^{44}-\mathrm{X}^{\mathrm{n}}-\mathrm{Phe}^{48}$ & $\mathrm{C}_{28} \mathrm{H}_{45} \mathrm{~N}_{7} \mathrm{O}_{7}$ & 591,70 & $-5,3$ & $\begin{array}{l}\mathrm{Glu}^{60}, \mathrm{Glu}^{62}, \mathrm{Thr}^{87}, \\
\mathrm{Ala}^{88}, \mathrm{Asp}^{89}, \mathrm{Ser}^{92}\end{array}$ \\
\hline $\begin{array}{l}\operatorname{Arg}^{131} *_{---} \text {Asp }^{61} \\
\text { (Standard 3) }\end{array}$ & $\begin{array}{l}\operatorname{Asp}^{131}-\mathrm{X}^{\mathrm{n}}- \\
\mathrm{Ile}^{133}\end{array}$ & $\mathrm{C}_{16} \mathrm{H}_{32} \mathrm{~N}_{6} \mathrm{O}_{4}$ & 372,46 & $-3,8$ & $\mathrm{Asp}^{61}, \mathrm{Glu}^{62}$ \\
\hline $\begin{array}{l}\text { Lys }^{140} *_{---} \text {Asp }^{61} \\
\text { Arg }^{143 *} *_{---G^{60}} \\
\text { (Standard 4) }\end{array}$ & $\begin{array}{l}\operatorname{Lys}^{140}-X^{\mathrm{n}}- \\
\operatorname{Arg}^{143}\end{array}$ & $\mathrm{C}_{27} \mathrm{H}_{46} \mathrm{~N}_{8} \mathrm{O}_{4}$ & 546,71 & $-5,13$ & $\mathrm{Asp}^{61}$ \\
\hline $\begin{array}{l}\text { Asn }^{147} *--\mathrm{Phe}^{93} \\
\text { His }^{114} ; \text { Arg }^{150 *} \text {--- } \\
\text { Glu }^{117}, \mathrm{Pro}^{203}, \mathrm{Ser}^{204} \\
\text { (Standard 5) }\end{array}$ & $\begin{array}{l}\operatorname{Asn}^{147}-\mathrm{X}_{-}- \\
\operatorname{Arg}^{150}\end{array}$ & $\mathrm{C}_{25} \mathrm{H}_{40} \mathrm{~N}_{8} \mathrm{O}_{5}$ & 532,64 & $-3,8$ & $\begin{array}{l}\mathrm{Phe}^{93}, \mathrm{Glu}^{117}, \mathrm{Pro}^{203}, \\
\mathrm{Ser}^{204}\end{array}$ \\
\hline 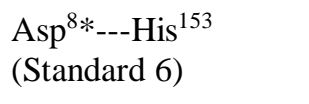 & Asp $^{8}$ dan $\operatorname{Ser}^{9}$ & $\mathrm{C}_{7} \mathrm{H}_{12} \mathrm{~N}_{2} \mathrm{O}_{5}$ & 204,18 & $-3,5$ & $\begin{array}{l}\mathrm{Ser}^{152}, \mathrm{His}^{153}, \mathrm{Glu}^{176}, \\
\mathrm{Ala}^{201}, \mathrm{Glu}^{202}, \mathrm{Ser}^{204}\end{array}$ \\
\hline $\begin{array}{l}\operatorname{Arg}^{14 *} *_{---L_{e u} 33} \\
\text { (Standard 7) }\end{array}$ & $\operatorname{Ser}^{13}-X^{\mathrm{n}}-\mathrm{Tyr}^{15}$ & $\mathrm{C}_{12} \mathrm{H}_{24} \mathrm{~N}_{4} \mathrm{O}_{4}$ & 288,34 & $-3,13$ & $\mathrm{Leu}^{33}, \mathrm{Ser}^{92}$ \\
\hline $\begin{array}{l}\operatorname{Arg}^{97} *_{---} \mathrm{Glu}^{34} \\
\text { Ser }^{100 *---S^{91}} \\
\text { (Standard 8) }\end{array}$ & $\begin{array}{l}\text { Asp }^{96}-X^{\mathrm{n}}- \\
\operatorname{Ser}^{100}\end{array}$ & $\mathrm{C}_{21} \mathrm{H}_{38} \mathrm{~N}_{6} \mathrm{O}_{8}$ & 502,56 & $-5,5$ & $\mathrm{Glu}^{34}, \mathrm{Ala}^{88}, \mathrm{Ser}^{91}$ \\
\hline 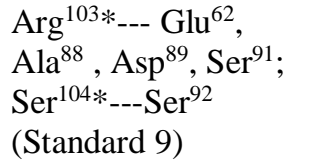 & $\begin{array}{l}\operatorname{Arg}^{103}-\mathrm{X}^{\mathrm{n}}- \\
\operatorname{Leu}^{105}\end{array}$ & $\mathrm{C}_{15} \mathrm{H}_{30} \mathrm{~N}_{6} \mathrm{O}_{4}$ & 358,44 & $-4,97$ & $\begin{array}{l}\mathrm{Glu}^{62}, \mathrm{Thr}^{87}, \mathrm{Ala}^{88}, \\
\text { Asps }^{89}, \mathrm{Thr}^{90}, \mathrm{Ser}^{91}, \\
\mathrm{Ser}^{92}\end{array}$ \\
\hline
\end{tabular}


Table 3 Bond type and atomic interaction between Epo and EpoR

\begin{tabular}{|c|c|c|c|c|c|}
\hline $\begin{array}{l}\text { Standards } \\
\text { Residues }\end{array}$ & $\begin{array}{l}\text { Epo-EpoR } \\
\text { Interaction }\end{array}$ & Bond Type & $\begin{array}{l}\text { Standards } \\
\text { Residues }\end{array}$ & $\begin{array}{l}\text { Interaction with } \\
\text { EpoR }\end{array}$ & $\begin{array}{l}\text { Type } \\
\text { interaction }\end{array}$ \\
\hline \multirow{4}{*}{$\begin{array}{l}\text { Standard 1 } \\
\left(\mathrm{Ala}^{19}-\mathrm{X}^{\mathrm{n}}-\right. \\
\left.\mathrm{Glu}^{23}\right)\end{array}$} & $\mathrm{H} \mathrm{Ala}^{19}-\mathrm{O} \mathrm{Glu}^{202}$ & Hidrogen & \multirow[t]{4}{*}{$\begin{array}{l}\text { Standard } 6\left(\mathrm{Asp}^{8}\right. \\
\left.\text { dan } \operatorname{Ser}^{9}\right)\end{array}$} & $\mathrm{O} \mathrm{Asp}^{8}-\mathrm{O} \mathrm{Ser}^{152}$ & $\begin{array}{l}\text { Van der } \\
\text { waals }\end{array}$ \\
\hline & $\mathrm{H} \mathrm{Ala}^{22}-\mathrm{O} \mathrm{Pro}^{203}$ & Hidrogen & & O Asp ${ }^{8}-\mathrm{H} \mathrm{His}^{153}$ & Hidrogen \\
\hline & $\mathrm{H} \mathrm{Glu}^{23}-\mathrm{O} \mathrm{Pro}^{203}$ & Hidrogen & & H Asp ${ }^{8}-\mathrm{O} \mathrm{Ser}^{152}$ & Hidrogen \\
\hline & $\begin{array}{l}\mathrm{O} \mathrm{Glu}^{23}-\mathrm{O} \mathrm{Glu}^{117} \\
\text { Pro }^{203} \text {, dan Ser }{ }^{204} \\
\mathrm{O} \mathrm{Glu}^{23}-\mathrm{H} \mathrm{Asn}^{116}\end{array}$ & $\begin{array}{l}\text { Van der } \\
\text { waals } \\
\text { Hidrogen }\end{array}$ & & $\begin{array}{l}\mathrm{OS} \mathrm{Ser}^{9}-\mathrm{O} \mathrm{Glu}^{176} \\
\mathrm{Ala}^{201}, \text { dan Glu }^{202} \\
\mathrm{O} \mathrm{Ser}^{9}-\mathrm{H} \mathrm{Ser}^{204}\end{array}$ & $\begin{array}{l}\text { Van der } \\
\text { waals } \\
\text { Hidrogen }\end{array}$ \\
\hline \multirow{9}{*}{$\begin{array}{l}\text { Standard } 2 \\
\left(T_{h r}^{44}-X^{n}-\right. \\
\left.\text { Phe }^{48}\right)\end{array}$} & $\begin{array}{l}\mathrm{O} \mathrm{Thr}^{44}-\mathrm{O} \mathrm{Glu}^{60} \\
\text { dan } \mathrm{Glu}^{62}\end{array}$ & $\begin{array}{l}\text { Van der } \\
\text { waals }\end{array}$ & & $\mathrm{H} \mathrm{Ser}^{9}-\mathrm{O} \mathrm{Glu}^{176}$ & Hidrogen \\
\hline & $\mathrm{H}_{\mathrm{Thr}}{ }^{44}-\mathrm{O} \mathrm{Glu}^{60}$ & Hidrogen & $\begin{array}{l}\text { Standard } 7 \\
\left(\operatorname{Ser}^{13}-X^{\mathrm{n}}-\mathrm{Tyr}^{15}\right)\end{array}$ & $\begin{array}{l}\mathrm{H} \mathrm{Ser}^{13}-\mathrm{O} \mathrm{Leu}^{33} \text { dan } \\
\mathrm{Ser}^{92}\end{array}$ & Hidrogen \\
\hline & $\mathrm{O} \mathrm{Thr}^{44}-\mathrm{H} \mathrm{Ser}^{91}$ & Hidrogen & \multirow[t]{4}{*}{$\begin{array}{l}\text { Standard } 8 \\
\left(\text { Lys }^{96}-X^{\mathrm{n}}-\text { Ser }^{100}\right)\end{array}$} & $\mathrm{H} \mathrm{Lys}^{97}-\mathrm{O} \mathrm{Glu}^{62}$ & Hidrogen \\
\hline & $\begin{array}{l}\mathrm{H} \mathrm{Lys}^{45}-\mathrm{O} \mathrm{Ala}^{88} \text {, } \\
\text { dan Asp }\end{array}$ & Hidrogen & & $\mathrm{H} \mathrm{Ala}^{98}-\mathrm{O} \mathrm{Thr}^{90}$ & $\begin{array}{l}\text { Van der } \\
\text { waals }\end{array}$ \\
\hline & $\mathrm{H} \mathrm{Val}^{46}-\mathrm{O} \mathrm{Ser}^{91}$ & Hidrogen & & $\begin{array}{l}\text { O Ser }{ }^{100}-\mathrm{O} \mathrm{Leu}^{33} \\
\text { dan Thr }\end{array}$ & $\begin{array}{l}\text { Van der } \\
\text { waals }\end{array}$ \\
\hline & $\mathrm{O} \mathrm{Va}{ }^{46}-\mathrm{O} \mathrm{Ser}^{92}$ & $\begin{array}{l}\text { Van der } \\
\text { waals }\end{array}$ & & O Ser ${ }^{100}-\mathrm{H} \mathrm{Ser}^{92}$ & Hidrogen \\
\hline & $\mathrm{H} \mathrm{Asn}^{47}-\mathrm{O} \mathrm{Thr}^{87}$ & Hidrogen & \multirow[t]{3}{*}{$\begin{array}{l}\text { Standard } 9 \\
\left(\text { Arg }^{103}-X^{\mathrm{n}_{-}}\right. \\
\left.\mathrm{Lue}^{105}\right)\end{array}$} & $\begin{array}{l}\mathrm{H} \mathrm{Arg}{ }^{103}-\mathrm{O} \mathrm{Glu}^{62}, \\
\mathrm{Ala}^{88}, \mathrm{Asp}^{89} \text {, dan } \\
\mathrm{Ser}^{91}\end{array}$ & Hidrogen \\
\hline & $\begin{array}{l}\mathrm{O} \mathrm{Asn}{ }^{47}-\mathrm{O} \mathrm{Ala}^{88} \\
\text { dan } \mathrm{Ser}^{11}\end{array}$ & $\begin{array}{l}\text { Van der } \\
\text { waals }\end{array}$ & & O Ser ${ }^{104}-\mathrm{H} \mathrm{Ser}^{92}$ & Hidrogen \\
\hline & $\mathrm{O} \mathrm{Phe}^{48}-\mathrm{H} \mathrm{Ser}^{92}$ & Hidrogen & & $\mathrm{H} \mathrm{Ser}^{104}-\mathrm{O} \mathrm{Ser}^{91}$ & Hidrogen \\
\hline \multirow{4}{*}{$\begin{array}{l}\text { Standard } 3 \\
\left(\text { Aspp }^{131}-\mathrm{X}^{\mathrm{n}}-\right. \\
\left.\mathrm{Ile}^{133}\right)\end{array}$} & $\mathrm{HArg}{ }^{131}-\mathrm{O} \mathrm{Glu}{ }^{62}$ & Hidrogen & & \multirow{9}{*}{$\begin{array}{l}\mathrm{O} \mathrm{Leu}^{105}-\mathrm{O} \mathrm{Ala}^{88} \\
\text { dan Ser } \\
\mathrm{O} \mathrm{Leu}^{105}-\mathrm{H} \mathrm{Thr}^{90} \\
\text { dan Ser }\end{array}$} & \multirow{9}{*}{$\begin{array}{l}\text { Van der } \\
\text { waals } \\
\text { Hidrogen }\end{array}$} \\
\hline & $\mathrm{H} \mathrm{Thr}^{132}-\mathrm{O} \mathrm{Asp}^{61}$ & Hidrogen & & & \\
\hline & $\mathrm{O} \mathrm{Thr}^{132}-\mathrm{O} \mathrm{Asp}^{61}$ & $\begin{array}{l}\text { Van der } \\
\text { waals }\end{array}$ & & & \\
\hline & $\mathrm{H} \mathrm{Ile}^{133}-\mathrm{O} \mathrm{Asp}{ }^{61}$ & Hidrogen & & & \\
\hline $\begin{array}{l}\text { Standard } 4 \\
\left(\operatorname{Lys}^{140}-X^{n_{-}}\right. \\
\left.\operatorname{Arg}^{143}\right)\end{array}$ & $\mathrm{H} \operatorname{Arg}^{143}-\mathrm{O} \mathrm{Asp}^{61}$ & Hidrogen & & & \\
\hline \multirow{4}{*}{$\begin{array}{l}\text { Standard } 5 \\
\left(\operatorname{Asn}^{147}-X^{n_{-}}\right. \\
\left.\operatorname{Arg}^{150}\right)\end{array}$} & $\mathrm{O} \mathrm{Asn}^{147}-\mathrm{O} \mathrm{Phe}^{93}$ & $\begin{array}{l}\text { Van der } \\
\text { waals }\end{array}$ & & & \\
\hline & O Phe ${ }^{148}-\mathrm{O} \mathrm{Ser}^{204}$ & $\begin{array}{l}\text { Van der } \\
\text { waals }\end{array}$ & & & \\
\hline & O Leu ${ }^{149}-$ O Pro ${ }^{203}$ & $\begin{array}{l}\text { Van der } \\
\text { waals }\end{array}$ & & & \\
\hline & $\begin{array}{l}\mathrm{H} \mathrm{Arg}^{150}-\mathrm{O} \mathrm{Glu}^{117} \text {, } \\
\text { dan } \text { Pro }^{203}\end{array}$ & Hidrogen & & & \\
\hline
\end{tabular}




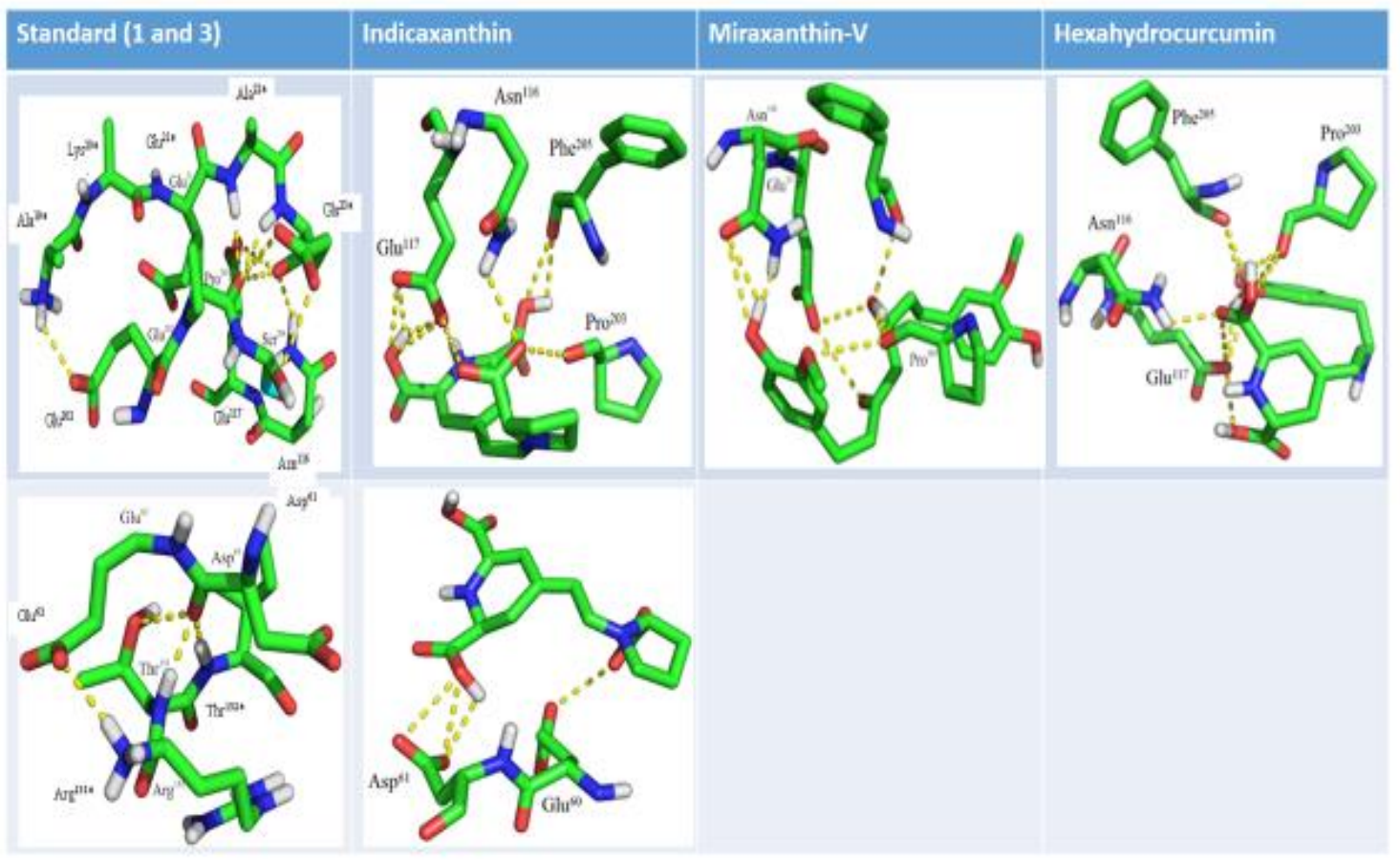

Fig. 1 Visualization of Epo standards (1 and 3)/phytochemicals and EpoR binding complexes using PyMol. Green: Carbon $(\mathrm{C})$, Red: Oxygen $(\mathrm{O})$, White: Hydrogen $(\mathrm{H})$, Blue: Nitrogen $(\mathrm{N})$, Yellow: Sulfur $(\mathrm{S})$, dashes-line: atomic interactions, *: Epo residues.

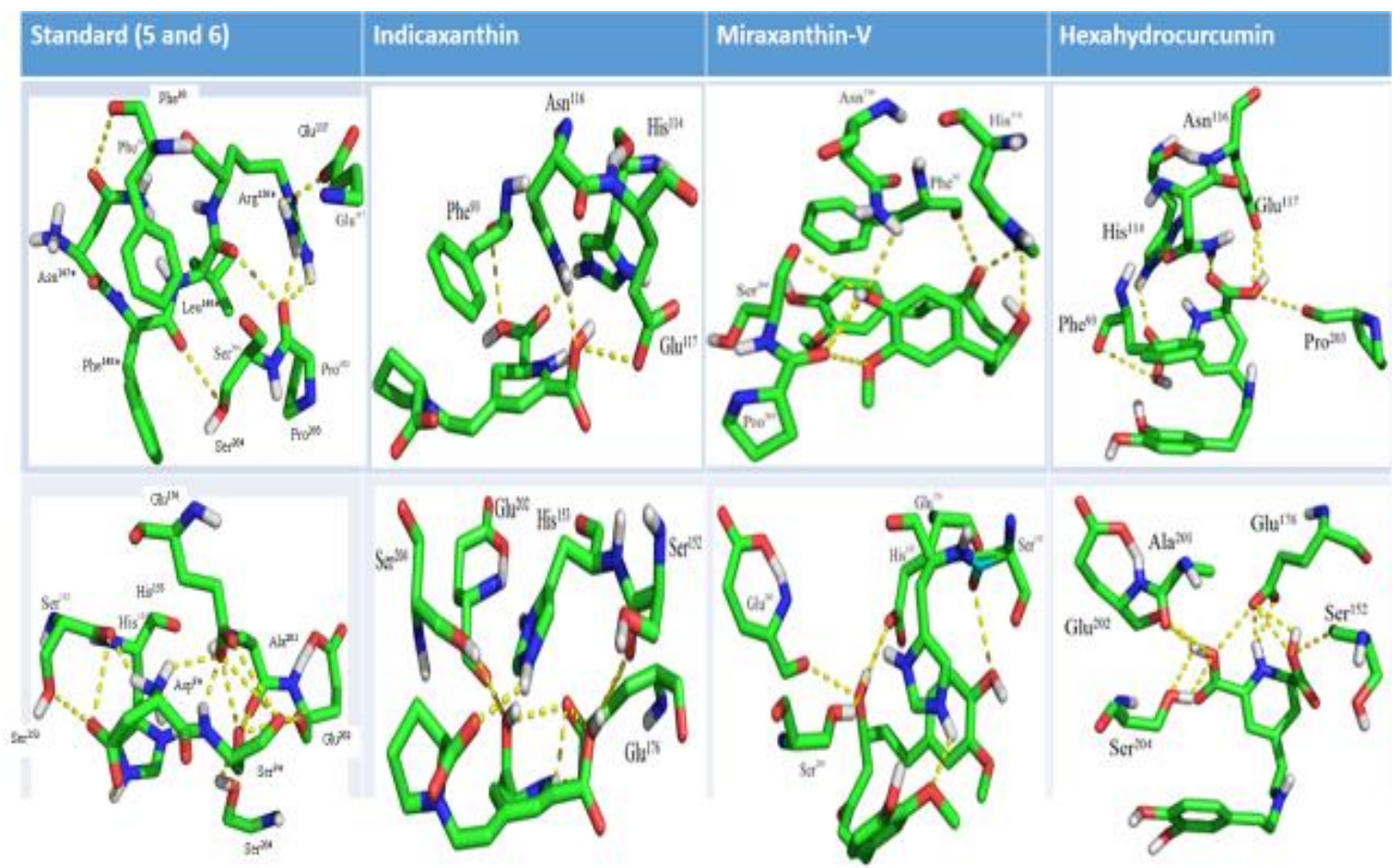

Fig. 2 Visualization of docking standards 5 and 6 and phytochemicals was performed using PyMol. Green: Carbon (C), Red: Oxygen (O), White: Hydrogen (H), Blue: Nitrogen (N), Yellow: Sulfur (S), dashes-line: atom interactions, *: Epo residues. 


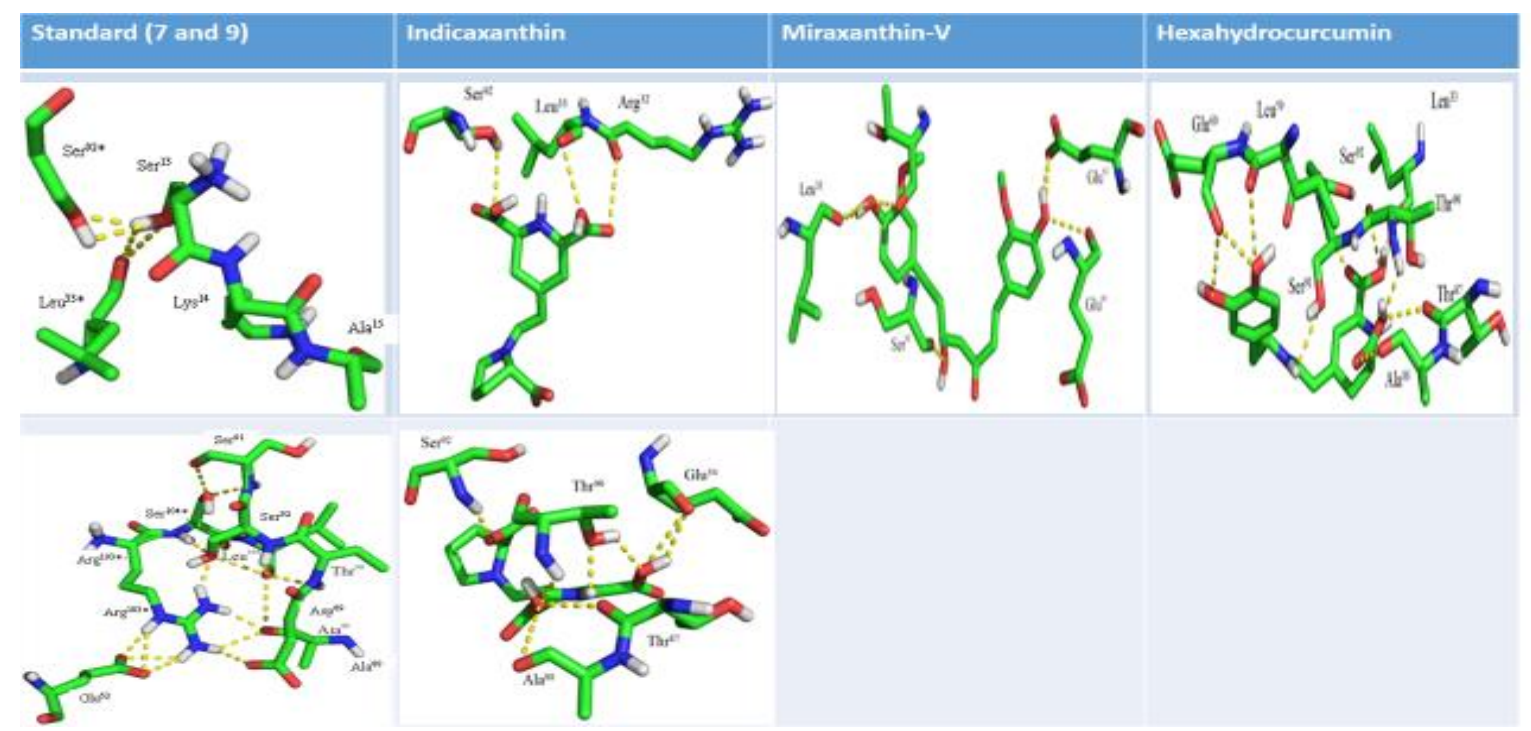

Fig. 3 Visualization of docking standards 7 and 9 and phytochemicals was performed using PyMol. Green: Carbon (C), Red: Oxygen (O), White: Hydrogen (H), Blue: Nitrogen (N), Yellow: Sulfur (S), dashes-line: atom interactions, *: Epo residues.

Total of 518 phytochemicals which was registered in HerbaldB and Pubchem met Lipinski's criteria. They were molecularly docked with EpoR and was evaluated their binding energy, location of interaction and molecule conformation. There were three phytochemicals (Indicaxanthin, Miraxanthin-V and Hexahydrocurcumin) which were similar with truncated Epo standards (table 4). They had similar molecular weight $( \pm 300 \mathrm{~g} / \mathrm{mol})$ and lipophilicity. Similar hydrogen acceptor and donor were found in Indicaxanthin and Hexahydrocurcumin while Miraxanthin- $\mathrm{V}$ had higher number of hydrogen acceptor and donor, compared with Indicaxanthin and Hexahydrocurcumin. These phytochemicals had lower binding energy than binding energy of truncated Epo standards. In terms of molecule interaction, Indicaxanthin had the same interaction with EpoR as the truncated Epo standards did while 11 molecule interaction was observed in Miraxanthin- $\mathrm{V}$ and Hexahydrocurcumin (table 4 and 5). All phytochemicals had hydrogen and van der walls bonds but Indicaxanthin and Hexahydrocurcumin were able to interact with $\mathrm{Phe}^{93}$ residue with van der walls bond.

Indicaxanthin, Miraxanthin- $\mathrm{V}$, and Hexahydrocurcumin have higher binding affinity than the standard Epo so that these phytochemicals can easily bind to EpoR. In addition, the phytochemicals have high similarity to the standard Epo. However, Indicaxanthin and Hexahydrocurcumin have an additional bond to $\mathrm{Phe}^{93}$ residue which is play important roles in hidrophobic nature and nonpolar interactions with EpoR (Middleton et al., 1998; Singh et al., 2012).

We have identified $10\left(\mathrm{Arg}^{32}, \mathrm{Leu}^{59}, \mathrm{Lys}^{65}, \mathrm{Thr}^{90}, \mathrm{Asn}^{116}, \mathrm{Met}^{150}, \mathrm{Ser}^{152}, \mathrm{Glu}^{176}, \mathrm{Ala}^{201}\right.$, and $\mathrm{Phe}^{205}$ ) additional binding sites of EpoR in all phytochemicals. Some studies have reported that $\mathrm{Met}^{150}$, Phe205, $\mathrm{Leu}^{59}$, $\mathrm{Thr}^{90}, \mathrm{Asn}^{116} \mathrm{Ser}^{152}$, and Glu ${ }^{176}$ residues contribute in EpoR binding. Met $^{150}$ and $\mathrm{Phe}^{205}$ residues are the most important amino acids for binding to EpoR of exogenous Epos like Epo-mimetic peptide (EMP1). Eventhough this compound has no homology to Epo, it binds specifically to the EpoR and mimics Epo biological effects (Middleton et al, 1998; Singh et al, 2012). It has reported that Lys ${ }^{65}$ amino acid facilitates EpoR to interact with Epo in loops $\mathrm{C}$ and $\mathrm{D}$ and $\mathrm{Asn}{ }^{116}$, a polar residue covers hydrophobic binding sites of EpoR to allow for their interaction (Barbone et al., 1997; Linvah et al., 1999). Therefore, the additional binding sites are required for bond stability of phytochemicals to EpoR.

Because Indicaxanthin, Miraxanthin- $\mathrm{V}$, and Hexahydrocurcumin have molecular weight less than 500 daltons, they can easily penetrate cell membrane of the human body. In 
addition, these phytochemicals have hydrogen donor $<5$, hydrogen acceptor $<10$, and high lipophilicity, which are potential as an alternative Epo agonist (Lipinski et al, 2001).

We have firstly demonstrated that Indicaxanthin, Miraxanthin-V, and Hexahydrocurcumin potentially become a natural Epo agonist in silico. Indicaxanthin and Miraxanthin-V have antioxidant activity. Exctract of both phytochemicals have been used for treatment of thalassemia patients which decrease perferryl-Hb. Both phytochemicals are found in Mirabilis japala roots and leaves which are used for treatment tonsillitis, cystysis, and leucorrhoea (Butera et al., 2002; Tesoriere et al., 2005; PubChem, 2017). A cytotoxic effect against colorectal cancer is detected in Hexahydrocurcumin which is found in Zingiber officinale (Srimuangwong et al., 2012; PubChem, 2017). A study has reported that Zingiber officinale extracts indicate biological effects such as immunomodulator, antitumor, antiinflammation, anti-apoptosis, anti-hyperglycemia, anti-lipidemia and anti-emetics (Ali et al., 2007).

In this study, we can not molecularly dock whole molecule of Epo with EpoR since AutoDock Vina program is unable to run high molecules ( $M W=>500 \mathrm{~g} / \mathrm{mol}$ ). This docking program is suitable for running rigid macromolecules and flexible ligands. It does not depict endogenous macromolecules that have flexible conformation. 
Table 4 Docking score and Lipinski's criteria of phytochemicals compared to truncated Epos as standards.

\begin{tabular}{|c|c|c|c|c|c|c|c|c|c|c|c|c|c|c|c|}
\hline \multirow{2}{*}{$\begin{array}{l}\text { Pubchem } \\
\text { ID }\end{array}$} & \multirow{2}{*}{ Ligand } & \multirow[b]{2}{*}{1} & \multirow[b]{2}{*}{2} & \multicolumn{7}{|c|}{ Mean Docking Score (kcal/mol) } & \multirow{2}{*}{$\begin{array}{l}\text { Molecular } \\
\text { chemical } \\
\text { formulas }\end{array}$} & \multicolumn{4}{|c|}{ Lipinski's Criteria } \\
\hline & & & & 3 & 4 & 5 & 6 & 7 & 8 & 9 & & $\begin{array}{c}\text { Molecular } \\
\text { weight }<500 \\
(\mathrm{~g} / \mathrm{mol})\end{array}$ & $\begin{array}{c}\mathrm{H}- \\
\text { Bond } \\
\text { Donor } \\
(<5)\end{array}$ & $\begin{array}{l}\text { H-Bond } \\
\text { Acceptor } \\
(<10)\end{array}$ & $\begin{array}{c}\text { Compound's } \\
\text { lipophilicity } \\
(\log \mathrm{P}<5)\end{array}$ \\
\hline & $\mathrm{Ala}^{19}-\mathrm{X}^{\mathrm{n}}-\mathrm{Glu}^{23}$ & -3.90 & - & - & - & - & - & - & - & - & $\mathrm{C}_{19} \mathrm{H}_{31} \mathrm{~N}_{5} \mathrm{O}_{9}$ & 473.48 & - & - & - \\
\hline & $\mathrm{Thr}^{44}-\mathrm{X}^{\mathrm{n}}-\mathrm{Phe}^{48}$ & - & -5.20 & - & - & - & - & - & - & - & $\mathrm{C}_{28} \mathrm{H}_{45} \mathrm{~N}_{7} \mathrm{O}_{7}$ & 591.70 & - & - & - \\
\hline & Asp $^{131}-\mathrm{X}^{\mathrm{n}}-\mathrm{Ile}^{133}$ & - & - & -2.60 & - & - & - & - & - & - & $\mathrm{C}_{16} \mathrm{H}_{32} \mathrm{~N}_{6} \mathrm{O}_{4}$ & 372.46 & - & - & - \\
\hline & $\begin{array}{l}\text { Lys }^{140}-X^{\mathrm{n}_{-}} \\
\text {Arg }^{143}\end{array}$ & - & - & - & -5.13 & - & - & - & - & - & $\mathrm{C}_{27} \mathrm{H}_{46} \mathrm{~N}_{8} \mathrm{O}_{4}$ & 546.71 & - & - & - \\
\hline & $\begin{array}{l}\operatorname{Asn}^{147}-X^{n_{-}} \\
\operatorname{Arg}^{150}\end{array}$ & - & - & - & - & -3.80 & - & - & - & - & $\mathrm{C}_{25} \mathrm{H}_{40} \mathrm{~N}_{8} \mathrm{O}_{5}$ & 532.64 & - & - & - \\
\hline & Asp $^{8}$ dan Ser 9 & - & - & - & - & - & -3.50 & - & - & - & $\mathrm{C}_{7} \mathrm{H}_{12} \mathrm{~N}_{2} \mathrm{O}_{5}$ & 204.18 & - & - & - \\
\hline & $\operatorname{Ser}^{13}-X^{\mathrm{n}}-\mathrm{Tyr}^{15}$ & - & - & - & - & - & - & -3.13 & - & - & $\mathrm{C}_{12} \mathrm{H}_{24} \mathrm{~N}_{4} \mathrm{O}_{4}$ & 288.34 & - & - & - \\
\hline & Lys $^{97}-X^{\mathrm{n}}-\mathrm{Ser}^{100}$ & - & - & - & - & - & - & - & -5.50 & - & $\mathrm{C}_{17} \mathrm{H}_{33} \mathrm{~N}_{5} \mathrm{O}_{5}$ & 387.47 & - & - & - \\
\hline & $\begin{array}{l}\operatorname{Arg}^{103}-X^{n_{-}} \\
\operatorname{Leu}^{105}\end{array}$ & - & - & - & - & - & - & - & - & -4.97 & $\mathrm{C}_{15} \mathrm{H}_{30} \mathrm{~N}_{6} \mathrm{O}_{4}$ & 358.44 & - & - & - \\
\hline 6096870 & Indicaxanthin & -4.00 & - & - & -6.50 & -4.50 & -4.00 & -3.67 & -5.53 & -5.50 & $\underline{\mathrm{C}_{14}} \mathrm{H}_{16} \mathrm{~N}_{2} \mathrm{O}_{6}$ & 308.29 & 3 & 7 & 0.4 \\
\hline 5281203 & Miraxanthin-V & -4.73 & - & - & - & -5.03 & -4.60 & -3.63 & -5.37 & -6.17 & $\underline{\mathrm{C}_{17} \mathrm{H}_{18} \mathrm{~N}_{2} \mathrm{O}_{6}}$ & 346.39 & 5 & 8 & 1.1 \\
\hline 5318039 & $\begin{array}{l}\text { Hexahydrocurc } \\
\text { umin }\end{array}$ & -3.93 & - & - & - & -4.37 & -4.00 & - & - & -5.00 & $\underline{\mathrm{C}_{21} \mathrm{H}_{26} \mathrm{O}_{6}}$ & 374.43 & 3 & 6 & 2.7 \\
\hline
\end{tabular}


Table 5 Location of phytochemicals interaction with EpoR monomer 1 (standards 1-5) and monomer 2 (standards 6-9) compared to truncated Epos

\begin{tabular}{|c|c|c|c|c|c|c|}
\hline \multirow[t]{2}{*}{ Pubchem ID } & \multirow[t]{2}{*}{ Ligand } & \multicolumn{5}{|c|}{ Location of interaction with standards } \\
\hline & & 1 & 2 & 3 & 4 & 5 \\
\hline & $\mathrm{Ala}^{19}-\mathrm{X}^{\mathrm{n}}-\mathrm{Glu}^{23}$ & $\mathrm{Glu}^{202}$ & - & - & - & - \\
\hline & $\mathrm{Thr}^{44}-\mathrm{X}^{\mathrm{n}}-\mathrm{Phe}^{48}$ & - & $\begin{array}{l}\mathrm{Glu}^{62}, \mathrm{Ser}^{92}, \mathrm{Thr}^{87} \text {, } \\
\text { Ala }^{88}, \mathrm{Phe}^{93}\end{array}$ & - & - & - \\
\hline & $\mathrm{Asp}^{131}-\mathrm{X}^{\mathrm{n}}-\mathrm{Ile}^{133}$ & - & - & $\mathrm{Asp}^{61}, \mathrm{Glu}^{62}$ & - & - \\
\hline & $\operatorname{Lys}^{140}-X^{\mathrm{n}}-\operatorname{Arg}^{143}$ & - & - & - & $\mathrm{Glu}^{60}, \mathrm{Asp}^{61}$ & - \\
\hline & $\operatorname{Asn}^{147}-X^{n}-\operatorname{Arg}^{150}$ & - & - & - & - & $\begin{array}{l}\mathrm{Phe}^{93}, \mathrm{His}^{114}, \mathrm{Glu}^{117} \text {, } \\
\mathrm{Ser}^{204}\end{array}$ \\
\hline 6096870 & Indicaxanthin & $\begin{array}{l}\text { Asn }^{116}, \text { Glu }^{117}, \\
\text { Pro }^{203}, \text { Phe }^{205}\end{array}$ & - & $\mathrm{Glu}^{60}, \mathrm{Asp}^{61}$ & & $\begin{array}{l}\mathrm{Phe}^{93}, \mathrm{His}^{114}, \mathrm{Asn}^{116}, \\
\mathrm{Glu}^{117}\end{array}$ \\
\hline 5281203 & Miraxanthin-V & $\begin{array}{l}\text { Asn }^{116}, \mathrm{Glu}^{117}, \\
\text { Pro }^{203}, \mathrm{Phe}^{205}\end{array}$ & - & - & - & $\begin{array}{l}\mathrm{Phe}^{93}, \mathrm{His}^{114}, \mathrm{Glu}^{117} \\
\text { Pro }^{203}, \mathrm{Phe}^{205}\end{array}$ \\
\hline 5318039 & Hexahydrocurcumin & $\begin{array}{l}\text { Asn }^{116}, \mathrm{Glu}^{117} \\
\text { Pro }^{203}, \mathrm{Phe}^{205}\end{array}$ & - & - & - & $\begin{array}{l}\mathrm{Phe}^{93}, \mathrm{His}^{114}, \mathrm{Asn}^{116}, \\
\mathrm{Pro}^{203}, \mathrm{Ser}^{204}\end{array}$ \\
\hline \multirow[t]{6}{*}{ Pubchem ID } & Ligand & \multicolumn{5}{|c|}{ Location of interaction with standards } \\
\hline & & 6 & 7 & 8 & 9 & \\
\hline & $\mathrm{Asp}^{8}$ dan $\mathrm{Ser}^{9}$ & $\begin{array}{l}\mathrm{Ser}^{152}, \mathrm{His}^{153} \\
\text { Glu }^{176}, \mathrm{Ala}^{201} \\
\text { Glu }^{202}, \mathrm{Ser}^{204}\end{array}$ & - & - & - & \\
\hline & $\operatorname{Ser}^{13}-X^{n}-T_{y r}{ }^{15}$ & - & $\mathrm{Leu}^{33}, \mathrm{Ser}^{92}$ & - & - & \\
\hline & $\operatorname{Lys}^{97}-X^{\mathrm{n}}-\mathrm{Ser}^{100}$ & - & - & $\begin{array}{l}\mathrm{Glu}^{34}, \mathrm{Thr}^{87}, \\
\text { Ala }^{88}, \mathrm{Thr}^{90}, \\
\text { Ser }^{91}\end{array}$ & - & \\
\hline & $\operatorname{Arg}^{103}-X^{\mathrm{n}}-$ Leu $^{105}$ & - & - & - & $\begin{array}{l}\mathrm{Glu}^{62}, \mathrm{Thr}^{87}, \\
\text { Ala }^{88}, \mathrm{Asp}^{89}, \\
\text { Thrr }^{90}, \mathrm{Ser}^{91}, \\
\text { Ser }^{92}\end{array}$ & \\
\hline 6096870 & Indicaxanthin & $\begin{array}{l}\mathrm{Glu}^{34}, \mathrm{Thr}^{87} \\
\mathrm{Ala}^{88}, \mathrm{Thr}^{90}\end{array}$ & $\mathrm{Arg}^{32}, \mathrm{Leu}^{33}, \mathrm{Ser}^{92}$ & $\begin{array}{l}\mathrm{Glu}^{34}, \mathrm{Thr}^{87}, \\
\mathrm{Al}^{88}, \mathrm{Thr}^{90}, \\
\mathrm{Ser}^{92}\end{array}$ & $\begin{array}{l}\mathrm{Glu}^{34}, \mathrm{Thr}^{87} \text {, } \\
\mathrm{Ala}^{88}, \mathrm{Thr}^{90}\end{array}$ & \\
\hline 5281203 & Miraxanthin-V & $\begin{array}{l}\text { Leu }^{33}, \text { Leu }^{59} \\
\text { Glu }^{60}, \text { Thr87, } \\
\text { Ala }^{88}, \text { Thrr }^{90} \\
\text { Ser }^{91}, \text { Ser }^{92}\end{array}$ & - & - & $\begin{array}{l}\text { Leu }^{33}, \text { Leu }^{59} \text {, } \\
\text { Glu }^{60}, \mathrm{Thr}^{87} \text {, } \\
\text { Ala }^{88}, \mathrm{Thr}^{90} \\
\text { Ser }^{91}, \mathrm{Ser}^{92}\end{array}$ & \\
\hline 5318039 & Hexahydrocurcumin & $\begin{array}{l}\text { Ser }^{152}, \text { His }^{153} \\
\text { Glu }^{176}, \text { Glu }^{202} \\
\text { Ser }^{204}\end{array}$ & - & - & $\begin{array}{l}\mathrm{Leu}^{33}, \mathrm{Glu}^{60}, \\
\mathrm{Glu}^{62}, \mathrm{Thr}^{90}, \\
\mathrm{Ser}^{92}\end{array}$ & \\
\hline
\end{tabular}

\section{CONCLUSION}

Indicaxanthin, Miraxanthin- $\mathrm{V}$, and Hexahydrocurcumin are potential as Epo agonist in silico to treat anemia in CKD. Further investigation should be done to verify these computational results.

\section{REFERENCE}

Ali, B.H., Blunden, G., Tanira, M.O., \& Nemmar A. (2007). Some phytochemical, pharmacological and toxicological properties of ginger (Zingiber officinale Roscoe): a review of recent research. Food Chem Toxicol. 46(2), 409-420. 
Balitbang Kemenkes RI. (2013). Riset Kesehatan Dasar; RISKESDAS 2013. Jakarta: Balitbang Kemenkes RI.

Barbone, F.P., Middleton, S.A., Johnson, D.L., McMahon, F.J., Tullai, J., Gruninger, R.H., .Schilling A.E. (1997). Mutagenesis studies of the human erythropoietin receptor Establishment of structure-function relationships. J Bio Chem, 272(8), 4985-4992.

Butera, D., Tesoriere, L., Di Gaudio, F., Bongiorno, A., Allegra, M., Pintaudi, A.M., \& Kohen, R. (2002). Antioxidant activities of sicilian prickly pear (opuntia ficus indica) fruit extracts and reducing properties of its betalains: betanin and indicaxanthin. J Agric Food Chem, 50 (23), 6895-6901.

Cases-Amenos, A., Castelao, A.M., Ros, J.F., Bartons, J.B., Ruiz, M.P., Prats, M.V., ,......Piera E.C. (2014). Prevalence of anemia and its clinical management in patients with stages 3-5 chronic kidney disease not on dialysis in Catalonia: MICENAS I study. Spanish Nephrol Soc, 34(2), 189-198.

Clement, F.M., Klarenbach, S., Tonelli, M., Johnson, J.A., \& Manns, B.S. (2009). The impact of selecting a high hemoglobin target level on health-related quality of life for patients with chronic kidney disease: A systematic review and meta-analysis. Arch Intern Med, 169(12), 1104-1112.

Ferreira, L.G., dos Santos, R.N., Oliva, G., \& Andricopulo, A.D. (2015). Molecular docking and structure-based drug design strategies. Molecules, 20(7), 13384-13421.

Garrido, P., Ribeiro, S., Fernandes, J., Vala, H., Pereira, P.R., Rocha, E.B., .....Belo L. (2012). Resistance to recombinant human erythropoietin therapy in a rat model of chronic kidney disease associated anemia. Int J Mol Sci, 8(24), 1206-1210.

Hayat A., Haria D., and Salifu M.O., 2008. Erythropoietin stimulating agents in the management of anemia of chronic kidney disease. Patient Prefer and Adher 2:195-200.

Johnson, D.W., Pollock, C.A., \& Macdougall, I.C. (2007). Erythropoiesis-stimulating agent hyporesponsiveness. Nephrol, 12(4), 321-330.

Kim, S.M., Kim, K.M., Kwon, S.K., \& Kim, H.Y. (2016). Erythropoiesis-stimulating agents and anemia in patients with non-dialytic chronic kidney disease. J Korean Med Sci. $31(1), 55-60$.

Linvah, O., Stura, E.A., Middleton, S.A., Johnson, D.L., Jolliffe, L.K., \& Wilson, I.A. (1999). Crystallographic evidence for preformed dimers of erythropoietin receptor before ligand activation. AAAS. 283(5404), 987-990.

Lipinski, C.A., Lombardo, F., Dominy, B.W., \& Feeney, P.J. (2001). Experimental and computational approaches to estimate solubility and permeability in drug discovery and development settings. Adc Drug Deliv Rev, 46(8), 3-26.

Macdougall, I.C., Bock, A., Carrera, F., Eckardt, K.U., Gaillard, C., Wyck, D.V., ....Roubert, B. 2014. The FIND-CKD study-a randomized controlled trial of intravenous iron versus oral iron in non-dialysis chronic kidney disease patients: background and rationale. Nephrol Dial Transplan, 29, 843-850.

Mehdi, U., \& Toto, R.D. (2009). Anemia, Diabetes, and Chronic Kidney Disease. Diabetes Care, 32(7), 1320-1321.

Middleton, S.A., Barbone, F.P., Johnson, D.L., Thurmond, R.L., You, Y., McMahon, F.J., ....Jin R. (1998). Shared and unique determinants of the erythropoietin (EPO) receptor are important for binding EPO and EPO mimetic peptide. JBC, 274(20), 14163-14169.

National Kidney Foundation (2012). Clinical Practice Guideline for Anemia in Chronic Kidney Disease. Kidney Int, 2(4), 279-335.

Oliveira, M.G., Delogo, K.N., Oliveira, H.M.G., Netto, A.R., Kritski, A.L., \& Oliveira, M.M. (2014). Anemia in hospitalized patients with pulmonary tuberculosis. J Bras Pneumol. 40(4), 403-410.

Palmer, S.C., Navaneethan, S.D., Craig, J.C., Johnson, D.W., Tonelli, M., Garg, A.X., Pellegrini, F., Ravani, P., Jardine, M., .....Perkovic, V. (2010). Meta-analysis: 
Erythropoiesis-stimulating agents in patients with chronic kidney disease. Ann Intern Med, 153(1), 23-33.

Palmer, S.C., Saglimbene, V., Mavridis, D., Salanti, G., Craig, J.C., Tonelli, M., ......Wiebe, N. (2014). Erythropoiesis-stimulating agents for anaemia in adults with chronic kidney disease: a network meta-analysis (Review). Ann Intern Med, 153(1), 23-33.

Parfrey, P.S., Lauve, M., Latremouille-Viau, D., \& Lefebvre, P. (2009). Erythropoietin therapy and left ventricular mass index in CKD and ESRD patients: A meta-analysis. Clin J Am Soc Nephrol, 4(4), 755-762.

PERNEFRI, 2014. $7^{\text {th }}$ Report of Indonesian Renal Registry. Indonesian Renal Registry. Retrieved from www.indonesianrenalregistry.org/data/INDONESIAN\%20RENAL\%20REGISTRY\% 202014.pdf.

PubChem. (2017). Indicaxanthin. National Center for Biotechnology Information. Retrieved from https://pubchem.ncbi.nlm.nih.gov/compound/6096870\#section=Top

PubChem. (2017). Miraxanthin- $V$. National Center for Biotechnology Information. Retrieved from https://pubchem.ncbi.nlm.nih.gov/compound/5281203\#section=Top

PubChem. (2017). Hexahydrocurcumin. National Center for Biotechnology Information. Retrieved from https://pubchem.ncbi.nlm.nih.gov/compound/Hexahydrocurcumin\#section=Top

Shestopalov, I..A., \& Zon, L.I. (2012). Drug discovery: Chemical beauty contest. Nature, 481(7382), 455-456.

Singh, V.K., Kumar, N., Kalsan, M., \& Saini, A. (2012). In silico designing and optimization of EPO mimetic using combinatorial library. IJSR, 3(12), 1110-1117.

Srimuangwong, K., Tocharus, C., Yoysungnoen, C.P., Suksamrarn, A., \& Tocharus J. (2012). Hexahydrocurcumin enhances inhibitory effect of 5-fluorouracil on HT-29 human colon cancer cells. World J Gastroenterol, 18 (19), 2383-2389.

Syed, R.S., Reid, S.W., Li, C., Cheetham, J.C., Aoki, K.H., Liu, B., .....Zhan, H. (1998). Efficiency of signaling through cytokine receptor depends critically on receptor orientation. Nature,395, 511-516.

Tang, Y.T., \& Marshall, G.R. (2011). Virtual screening for lead discovery. Springer Link. $716(1), 225-243$.

Tesoriere, L., Allegra, M., Butera, D., Gentile, C., \& Livrea M. A. (2005). Cytoprotective effects of the antioxidant phytochemical indicaxanthin in $\beta$-thalassemia red blood cells. Free Radic Res, 40(7), 753-761.

WHO. (2015). The global prevalence of anaemia in 2011. Geneva: World Health Organization.

Yanuar, A., Suhartanto, H., Mun'im, A., Anugraha, B.H., \& Syahdi R.R. (2012). Virtual screening of Indonesian herbal database as HIV-1 reverse transcriptase inhibitor. Bioinformation, 8(24), 1206-1210. 